\title{
Bayesian Tracking of Tubular Structures and Its Application to Carotid Arteries in CTA
}

\author{
Michiel Schaap, Rashindra Manniesing, Ihor Smal, \\ Theo van Walsum, Aad van der Lugt, and Wiro Niessen \\ Biomedical Imaging Group Rotterdam, \\ Departments of Radiology and Medical Informatics \\ Erasmus MC - University Medical Center Rotterdam \\ michiel.schaap@erasmusmc.nl
}

\begin{abstract}
This paper presents a Bayesian framework for tracking of tubular structures such as vessels. Compared to conventional tracking schemes, its main advantage is its non-deterministic character, which strongly increases the robustness of the method. A key element of our approach is a dedicated observation model for tubular structures in regions with varying intensities. Furthermore, we show how the tracking method can be used to obtain a probabilistic segmentation of the tracked tubular structure. The method has been applied to track the internal carotid artery from CT angiography data of 14 patients (28 carotids) through the skull base. This is a challenging problem, owing to the close proximity of bone, overlap in intensity values of lumen voxels and (partial volume) bone voxels, and the tortuous path of the vessels. The tracking was successful in 25 cases, and the extracted path were found to be close $(<1.0 \mathrm{~mm})$ to manually traced paths by two observers.
\end{abstract}

Keywords: Bayesian tracking, Elongated structures, Bhattacharrya metric, Carotid arteries.

\section{Introduction}

Segmentation and tracking of tubular elongated structures is an important goal in a wide range of biomedical imaging applications. Especially vessel tracking in medical images has received considerably attention, as it can e.g. be used as a preprocessing step towards the detection and characterization of vessel pathology, such as stenoses. In this work we present a new vessel tracking method which is based on a probabilistic approach.

Until recently almost all vessel tracking methods were deterministic in their approach; only one assumption of the track configuration is taken into account during tracking, e.g. 112]. The path or segmentation is found by updating the track to the most probable configuration at each iteration of the method. This may lead to incorrect tracking results because the path direction can not be derived from imaging data locally, e.g. owing to pathologies, corrupted or missing data. By taking into account multiple hypotheses during tracking this problem 
can be circumvented. Probabilistic methods for tracking elongated structures have been presented in a number of recent papers 34. These methods search globally for the path that reflects the priors as good as possible.

One of the important considerations when developing a tubular structure tracking algorithm is an observation model which describes the appearance of the tubular structures in the image, given the parameters of the tube (e.g. position and orientation). The two mentioned probabilistic approaches 34 use an observation model that is based on the assumption that vessels are bright relative to their background. This model is not suited for situations where the background contains both lower and higher intensities than the tube, which is e.g. the case for carotid arteries, which are surrounded by low intensity soft tissue and high intensity bone. This article is based on the method presented in 4. The novelty of this new work is threefold. First, we present a generic and intuitive observation model to overcome the problems associated with the bright vessel observational model. The new observation model is specifically tailored for tracking homogeneous tubular structures through backgrounds with varying intensities. Second, the article presents a method to reduce the possibility of tracking sections of tubular structures multiple times. Third, the Bayesian tracking methodology is used to obtain a rough segmentation of the vessel.

The presented method is evaluated by tracking the internal carotid arteries through the skull base, which is a challenging problem. The success rate and accuracy of the tracking are assessed quantitatively by comparing results on 14 datasets (28 carotids) to tracings by two observers.

\section{Bayesian Tracking of Tubular Structures}

This section presents our Bayesian tracking approach. For a reference of the methods and notations used in this article we would like to refer to the work of Doucet et al. 5. In Bayesian tracking, the algorithm searches for the track that is best explained by the data, given an observation model (which is based on our model of a tube), and prior information on the shape and appearance of the vessel (modeled via e.g. transition priors). The posterior probability density function of a track, given the data, is recursively estimated. This is achieved by adding new hypotheses to a set of currently most likely hypotheses. At the end, the maximum of the posterior probability is chosen as the track that best represents the vessel. In the following, we first describe our model of a tube, and how that model is implemented in our observation model. Next, it is explained how the posterior probability density can be estimated ('prediction'), and lastly the mechanism for generating new hypotheses, is explained ('update').

\subsection{Tube Model}

Our tracking method will consider a tube as a series of tube segments. A tube segment at iteration $t$ is described by its location $\boldsymbol{p}_{t}=\left(x_{t}, y_{t}, z_{t}\right)^{T}$, orientation $\boldsymbol{v}_{t}=\left(\theta_{t}, \phi_{t}\right)$, radius $r_{t}$, intensity $I_{t}$ and intensity variance $\sigma_{t}$. Thus each tube 
segment is characterized by a state vector $\mathbf{x}_{t}=\left(\boldsymbol{p}_{t}, \boldsymbol{v}_{t}, r_{t}, I_{t}, \sigma_{t}\right)^{T}$. This results in a tube configuration described by $\mathbf{x}_{0: t} \triangleq\left\{\mathbf{x}_{0}, \ldots, \mathbf{x}_{t}\right\}$, see Figure $1(\mathrm{a})$.

With every tube segment we associate a region of interest (ROI) $U$, defined by the components $\boldsymbol{p}_{t}, \boldsymbol{r}_{t}$, and $\boldsymbol{v}_{t}$ of $\mathbf{x}_{t}$ (see Fig. 1b). Subsequently, we let $\mathbf{z}_{t}$ denote the image measurements (i.e. image intensities) within this ROI. Hence, all measurements corresponding to tube $\mathbf{x}_{0: t}$ are denoted with $\mathbf{z}_{0: t}$.

$S\left(\mathbf{x}_{t}\right)$ defines the set of spatial coordinates that lie within the hypothesized tube and $B\left(\mathbf{x}_{t}\right)=U \backslash S$ defines the set of spatial coordinates and in the band around the tube, see Figure 1(b).

\subsection{Observation Model}

With $p\left(I \mid S\left(\mathbf{x}_{t}\right)\right)$ and $p\left(I \mid B\left(\mathbf{x}_{t}\right)\right)$ we describe the normalized intensity histograms of the voxels at the inside and outside of the tube segment respectively. These distributions are constructed by sampling, with nearest neighbor interpolation, from the ROI defined by $\mathbf{x}_{t}$.

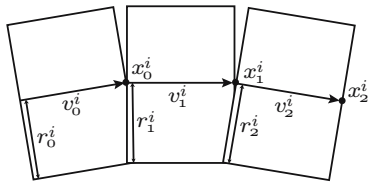

(a)

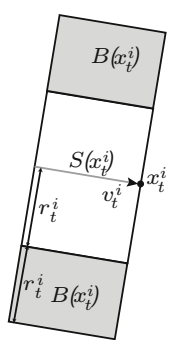

(b)

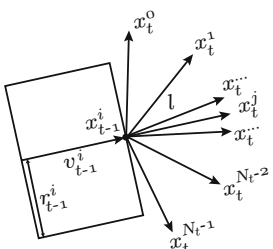

(c)

Fig. 1. Fig. (a) shows a part of the tube configuration $\mathbf{x}_{0: t}$. Fig. (b) visualizes the region in the tube $S\left(x_{t}^{i}\right)$ and the region in the band $B\left(x_{t}^{i}\right)$ around the tube. The prediction of new tubular segments, as explained in Section 2.3 is presented in Fig. (c).

In our observation model, we assume that tubes have a homogeneous intensity with additive Gaussian disturbance, which differs from the intensity of surrounding tissue. Given the tube segment $\mathbf{x}_{t}$ and the measurements $\mathbf{z}_{t}$, the likelihood of the observation given the state $p\left(\mathbf{z}_{t} \mid \mathbf{x}_{t}\right)$ is given by

$$
p\left(\mathbf{z}_{t} \mid \mathbf{x}_{t}\right) \propto p\left(\mathbf{z}_{t} \mid \boldsymbol{p}_{t}, \boldsymbol{v}_{t}, r_{t}, I_{t}, \sigma_{t}\right)=D_{c p, t}\left(1-D_{s b, t}\right) p_{M}\left(\mathbf{x}_{t}\right) p\left(r_{t}\right)
$$

where $D_{c p, t}$ and $D_{s b, t}$ denote the similarity between the intensity distributions of the current and the previous segment and the similarity between the intensity distribution of the inside and outside of the tube, respectively. Furthermore $p_{M}\left(\mathbf{x}_{t}\right)$ is a spatial prior that is used to prevent loops in the tracked tube and $p\left(r_{t}\right)$ is a user-defined and application specific prior for the expected radii.

The two intensity measures are calculated as follows

$$
\begin{aligned}
D_{c p, t} & =D\left(\mathcal{N}\left(I \mid \hat{I}_{s, t}, \hat{\sigma}_{s, t}^{2}\right), p\left(I \mid I_{t}, \sigma_{t}^{2}\right)\right)^{c_{1}} \\
D_{s b, t} & =D\left(\mathcal{N}\left(I \mid \hat{I}_{s, t}, \hat{\sigma}_{s, t}^{2}\right), p\left(I \mid B\left(\mathbf{x}_{t}\right)\right)\right)^{c_{2}},
\end{aligned}
$$


where $\mathcal{N}\left(. \mid \mu, \sigma^{2}\right)$ indicates a normal distribution with the mean $\mu$ and variance $\sigma^{2}, \hat{I}_{s, t}$ and $\hat{\sigma}_{s, t}^{2}$ describe the mean and variance of the histogram $p\left(I \mid S\left(\mathbf{x}_{t}\right)\right)$, and $c_{1}$ and $c_{2}$ are parameters to regulate the influence of the different components.

Several methods have been suggested in literature to calculate the similarity between two distributions. We use the Bhattacharyya Metric (BM) [6], defined by

$$
D\left(p_{1}, p_{2}\right)=B M\left(p_{1}, p_{2}\right)=\int \sqrt{p_{1}(x) p_{2}(x)} d x
$$

For the prior $p_{M}\left(\mathbf{x}_{t}\right)$ we store for each voxel the probability that the voxel has not been identified as being part of the tubular structure in a spatial map $M_{t}$, with $M_{0}=1$. This map is updated after each iteration. The update step is described in section 2.5. The prior is constructed by averaging over all values of this map that fall within the ROI described by $S\left(\mathbf{x}_{t}\right)$.

$$
p_{M}\left(\mathbf{x}_{t}\right)=\frac{\sum_{\boldsymbol{p} \in S\left(\mathbf{x}_{t}\right)} M_{t}(\boldsymbol{p})}{\left|S\left(\mathbf{x}_{t}\right)\right|},
$$

where |.| defines the set size operator. This prior serves two purposes. First, it is less likely that sections of the image are tracked twice. Second, this map presents a rough tube segmentation.

\subsection{Prediction}

Using the Bayesian rule, the pdf $p\left(\mathbf{x}_{0: t} \mid \mathbf{z}_{0: t}\right)$, that describes the posterior probability of the tube configuration, having all the observations up to iteration $t$, can be estimated with the following recursion $[5$

$$
p\left(\mathbf{x}_{0: t} \mid \mathbf{z}_{0: t}\right) \propto p\left(\mathbf{x}_{t} \mid \mathbf{x}_{t-1}\right) p\left(\mathbf{z}_{t} \mid \mathbf{x}_{t}\right) p\left(\mathbf{x}_{0: t-1} \mid \mathbf{z}_{0: t-1}\right),
$$

where the transition prior $p\left(\mathbf{x}_{t} \mid \mathbf{x}_{t-1}\right)$ is assumed to be Markovian ( $\mathbf{x}_{t}$ only depends on $\mathbf{x}_{t-1}$ and not on any other past states) and is factorized as

$$
p\left(\mathbf{x}_{t} \mid \mathbf{x}_{t-1}\right) \propto p\left(\boldsymbol{p}_{t}, \boldsymbol{v}_{t} \mid \boldsymbol{p}_{t-1}, \boldsymbol{v}_{t-1}\right) p\left(r_{t} \mid r_{t-1}\right),
$$

assuming that there is no transition model for $I_{t}$ and that all intensities are equally probable.

The likelihood $p\left(\mathbf{z}_{t} \mid \mathbf{x}_{t}\right)$ relates the conditionally independent measurements at iteration $t$ to the state $\mathbf{x}_{t}$, as defined in equation (1). At each iteration step, we represent the probability of the tube configuration with a set of $N_{t}$ weighted states $\boldsymbol{X}_{0: t}=\left\{\mathbf{x}_{0: t}^{i}, w_{t}^{i}\right\}_{i=1}^{N_{t}}$, thus

$$
p\left(\mathbf{x}_{0: t} \mid \mathbf{z}_{0: t}\right)=\sum_{i=1}^{N_{t}} w_{t}^{i} \delta\left(\mathbf{x}_{0: t}-\mathbf{x}_{0: t}^{i}\right),
$$

where $\delta(\cdot)$ is the Dirac delta function and the weights are normalized such that $\sum_{i=1}^{N_{t}} w_{t}^{i}=1$. 
In each iteration we use the variance of the weights $w_{t}^{i}$ to determine which hypotheses should be kept in the next iteration. The $N_{e}$ most probable hypotheses are kept according to the weights $w_{t}^{i}, i \in\left\{1, \ldots, N_{t}\right\}$, where

$$
N_{e}=\frac{1}{\sum_{i=1}^{N_{t}}\left(w_{t}^{i}\right)^{2}}
$$

From each of these states, $N_{t}^{i}=\max \left(\operatorname{nint}\left(w_{t}^{i} N\right), N_{\max }\right)$ new states are created, where nint(.) denotes a nearest integer round, $N$ is pre-defined and describes the maximum total number of hypotheses created and $N_{\max }$ describes the maximum number of hypotheses created from one hypothesis. This approach will keep only the relevant hypotheses and effectively distribute them according to the described pdf.

\section{$2.4 \quad$ Update}

In the update step, prior knowledge on the curvature of the centerline, the variance of the tube radius along the centerline, the intensity variance in the tube and the contrast-to-noise ratio in the image are incorporated. This is achieved as follows.

The formation of the $N_{t}^{i}$ new hypotheses $\mathbf{x}_{t}^{j}, j=\left\{0, \ldots, N_{t}^{i}-1\right\}$, at iteration $t$ from the previous hypothesis $\mathbf{x}_{t-1}^{i}$ consist of a transition to a new position $\boldsymbol{p}_{t}$, which is deterministically defined by

$$
\boldsymbol{p}_{t}^{j}=\boldsymbol{p}_{t-1}^{i}+R_{z}\left(\theta_{t-1}\right) R_{y}\left(\phi_{t-1}\right) R_{z}\left(\vartheta_{t}^{j}\right) R_{y}\left(\varphi_{t}^{j}\right)(0,0, l)^{T}
$$

where $R_{z}$ (.) and $R_{y}($.$) are rotation matrices around the z$-and $y$-axis [7. The length of a tube segment $l$ is user-defined. This parameter has influence on the propagation speed, the curvature of the track, and the possible contrast-to-noise ratio of the tube.

Figure 1(c) gives a schematic explanation of the transition in (10). The angles $\left(\vartheta_{t}^{j}, \varphi_{t}^{j}\right)$ describe a point in the local spherical coordinate system with the $z$-axis orientated in the direction of $\boldsymbol{v}_{t-1}^{i}$ and origin at $\boldsymbol{p}_{t-1}$. Therefore, the angle $\varphi_{t}^{j}$ is equal to the enclosed angle between $\boldsymbol{v}_{t-1}$ and $\boldsymbol{v}_{t}$. The two angles $\left(\vartheta_{t}^{j}, \varphi_{t}^{j}\right)$ are constructed with an algorithm that uniformly distributes points on a sphere, as described by Saff and Kuijlaars [8]. This algorithm is used to distribute the $N_{t}^{i}$ new hypotheses uniformly on the half sphere in front of $\boldsymbol{p}_{t-1}$ oriented in the direction of $\boldsymbol{v}_{t-1}$. In this case, the transition density $p\left(\boldsymbol{p}_{t}, \boldsymbol{v}_{t} \mid \boldsymbol{p}_{t-1}^{i}, \boldsymbol{v}_{t-1}^{i}\right)$ is given by

$$
p\left(\boldsymbol{p}_{t}, \boldsymbol{v}_{t} \mid \boldsymbol{p}_{t-1}^{i}, \boldsymbol{v}_{t-1}^{i}\right)=\sum_{j=1}^{N_{t}^{i}} \tilde{\omega}_{j} \delta\left(\boldsymbol{p}_{t}-\boldsymbol{p}_{t}^{j}\right)
$$

where the weight $\tilde{\omega}_{j}$ of a given enclosed angle is given by

$$
\begin{gathered}
\tilde{\omega}_{j}=\frac{\omega\left(\phi_{j}\right)}{\sum_{k=1}^{N_{t}^{i}} \omega\left(\phi_{k}\right)} \\
\omega(\varphi)=N\left(\varphi \mid 0, \sigma_{\varphi}^{2}\right)
\end{gathered}
$$

with $\sigma_{\varphi}$ being a pre-defined parameter. 
The transition of $r_{t}$ is described by $r_{t}=r_{t-1}+\eta_{t}$, where $\eta_{t}$ is an uncorrelated Gaussian random variable with variance $\sigma_{r}^{2}$. In this case $p\left(r_{t} \mid r_{t-1}\right)=$ $\mathcal{N}\left(r_{t} \mid r_{t-1}, \sigma_{r}^{2}\right)$. We estimate the radius of the patch $r_{t}^{j}$ as follows

$$
\hat{r}_{t}^{j}=\underset{r_{t}}{\operatorname{argmax}} p\left(\mathbf{z}_{t} \mid \boldsymbol{p}_{t}^{j}, \phi_{t}^{j}, r_{t}, I_{t \mid t-1}\right) p\left(r_{t} \mid r_{t-1}\right) .
$$

Then, the intensity and variance variables of the state vector are updated according to $I_{t}^{i}=\hat{I}_{s, t-1}^{i}$ and $\sigma_{t}^{i}=\hat{\sigma}_{s, t-1}^{i}$.

\subsection{Tube Probability Update}

At each iteration we calculate for each voxel the probability that it has been identified as being part of the tube with $P_{t}(\boldsymbol{p})=\sum_{\forall i: \boldsymbol{p} \in S\left(x_{t}^{i}\right)} w_{t}^{i}$.

$P_{t}$ is then used to update the map $M_{t}$ according to $M_{t}=M_{t-1}\left(1-P_{t}\right)$

\section{Carotid Arteries Through Skull Base}

The method is evaluated by tracking the internal carotid arteries (ICAs) in CTA data covering the anatomical region containing the skull base. This is a challenging problem because of the small spatial separation and strong overlap in intensity values of the ICAs and the skull base. A standard approach is the use of an additional CT scan to mask high intensity structures, but this is associated with increased radiation dose and increased acquisition time for the patient. Recently, methods have appeared that directly track the vessels in CTA, 91011, but either lack methodological details [9] and/or an extensive validation [10[11]. The method described in [11] seems to be the most promising to date, but evaluation was limited to visual inspection by observing whether the tracked path was fully contained within the ICAs. In this work we quantitatively compare the results of our method to manually traced paths by two observers.

\subsection{Data Acquisition and Parameter Settings}

Fourteen consecutive patients suffering from a transient ischemic attack underwent a CTA examination. The data, acquired on a 16-slice CT scanner (Siemens Somatom Sensation 16, Forchheim, Germany) were reconstructed using a B46f kernel, and subsampled in-plane with linear interpolation to a $256 \times 256$ matrix, resulting in voxel sizes of $0.5 \times 0.5 \times 1.2 \mathrm{~mm}$, in order to reduce the computational costs.

Visual inspection showed that 24 out of 28 carotids contained some form of pathology, 16 carotids showed mild calcifications, stenoses or aneurysms and eight carotids contained severe calcifications and/or stenoses.

Parameters of the method were fixed and empirically selected: $N=1000$, $N_{\max }=50, c=10, s=30, \sigma_{\varphi}^{2}=2.0, \sigma_{r}^{2}=0.5, p\left(r_{t}\right)=\mathcal{N}\left(r_{t} \mid 2.5 \mathrm{~mm}, 0.5 \mathrm{~mm}^{2}\right)$, and $l=4.5 \mathrm{~mm}$. 


\subsection{Evaluation Method}

The algorithm is initialized by placing a small vector in the vessel just after the carotid bifurcation. The method then iterates until a vessel length of at least 180 $\mathrm{mm}$ is tracked to ensure that each tracked path goes beyond the region containing the skull base and reaches the Circle of Willis. The segment length is $4 \mathrm{~mm}$, therefore the final path will contain 46 points. For comparison, two observers manually traced both ICAs in each patients (resulting in a total of 56 manually traced paths). Points were first clicked on the axial slices and then visually inspected on the MPR images to determine whether the point was properly centralized. Points could be corrected on the MPR images if needed.

A comparison between two paths is made segment-wise, by finding the minimum distances of two neighboring points on the first path to the second path. The radii of ICAs are approximately in the range of $1.0 \mathrm{~mm}-2.5 \mathrm{~mm}$. If both distances are found to be smaller than $2.5 \mathrm{~mm}$, than this path segment is considered to be corresponding. For the corresponding path segments the average distance is computed. In this way we both evaluate the tracking success rate and its accuracy. Both criteria are applied in the path comparisons between the method and observers $\mathcal{M} \rightarrow \mathcal{O}_{\{1,2\}}$, and between the observers $\mathcal{O}_{1} \leftrightarrow \mathcal{O}_{2}$.

\section{Results}

The results are summarized in Table 1 and 2. An example of the results is shown in figure 2. Overall, $95 \%$ of the tracked segments was found within $2.5 \mathrm{~mm}$ of the annotated centerlines and for these segments the average distance was approximately $0.7 \mathrm{~mm}$, which is $0.2 \mathrm{~mm}$ larger than the interobserver variability. Furthermore, the average distance of the corresponding segments per track was always smaller than $1.0 \mathrm{~mm}$.

Seven tracks had an overlap of less than $100 \%$ with the track of one of the observers. Visual inspection showed that an annotation error was made by observer two, causing one of these cases. The other six tracks with less than $100 \%$ correspondance can be divided in a group of three $(>80 \%)$ where the track has some minor localization errors in the upper parts of the ICA and a group of three $(<80 \%)$ where the method failed to track from the seedpoint to the Circle of Willis. For these vessels, respectively 57, 72 and $78 \%$ was tracked.

Table 1. The results of the comparison between observer $1\left(O_{1}\right)$, observer $1\left(O_{1}\right)$, and the method (M). 'Overlap' shows the percentage of the total length of all 14 tracks found within $2.5 \mathrm{~mm}$. 'Avg. Dist.' shows for all corresponding segments the average distance in millimeters to the reference track, weighted by segment length.

\begin{tabular}{l|c|c|c|c} 
& $\mathcal{M} \rightarrow \mathcal{O}_{1}$ & $\mathcal{M} \rightarrow \mathcal{O}_{2}$ & $\mathcal{O}_{1} \rightarrow \mathcal{O}_{2}$ & $\mathcal{O}_{2} \rightarrow \mathcal{O}_{1}$ \\
\hline Overlap & $0.95 \%$ & $0.95 \%$ & $0.99 \%$ & $0.99 \%$ \\
Avg. Dist. & 0.69 & 0.67 & 0.46 & 0.47
\end{tabular}

Table 2. The automatic tracks (M) categorized on the percentage found within $2.5 \mathrm{~mm}$ of the reference standard, respectively observer $1\left(\mathrm{O}_{1}\right)$ and $2\left(\mathrm{O}_{2}\right)$

\begin{tabular}{r|c|c} 
& $\mathcal{M} \rightarrow \mathcal{O}_{1}$ & $\mathcal{M} \rightarrow \mathcal{O}_{2}$ \\
\hline $100 \%$ & 22 & 22 \\
$90-100 \%$ & 1 & 2 \\
$80-90 \%$ & 2 & 3 \\
$<80 \%$ & 3 & 2
\end{tabular}




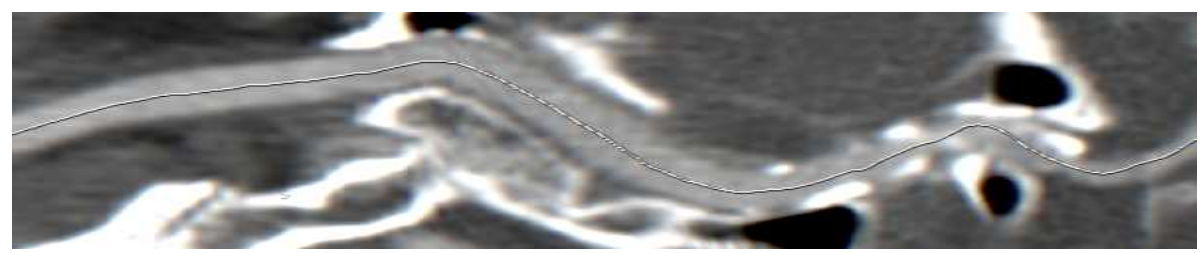

Fig. 2. An example of an automatically generated curved planar reformatted image. The image shows calcifications in the internal carotid artery in the region after the skull base, depicted on the right side of the image.

\section{Discussion and Conclusion}

In this paper we have presented a tubular tracking approach within a Bayesian framework. This approach has a number of advantages over most existing tracking approaches. First, the method is flexible, since prior information can easily be incorporated. In our case, important prior information is incorporated through the observation model. Second, its non-deterministic character increases robustness of the method.

The method has successfully been applied to the tracking of 28 ICAs through the difficult region of the skull base. The resulting paths were found to be close $(<1.0 \mathrm{~mm})$ to manually traced paths by two observers. Because the average computation time was approximately four minutes per carotid artery we believe that the method has large potential for clinical practice.

\section{References}

1. Aylward, S., Bullit, E.: Initialization, noise, singularities, and scale in height ridge traversal for tubular object centerline extraction. IEEE Transactions on Medical Imaging 21(2), 61-75 (2002)

2. Lorigo, L.M., Faugeras, O.D., Grimson, W.E.L., Keriven, R., Kikinis, R., Nabavi, A., Westin, C.-F.: Curves: Curve evolution for vessel segmentation. Medical Image Analysis 5, 195-206 (2001)

3. Florin, C., Paragios, N., Williams, J.: Globally optimal active contours, sequential monte carlo and on-line learning for vessel segmentation. In: Leonardis, A., Bischof, H., Pinz, A. (eds.) ECCV 2006. LNCS, vol. 3951, pp. 476-489. Springer, Heidelberg (2006)

4. Schaap, M., Smal, I., Metz, C., van Walsum, T., Niessen, W.: Bayesian tracking of elongated structures in 3d images. In: Karssemeijer, N., Lelieveldt, B. (eds.) IPMI 2007. LNCS, vol. 4584, pp. 74-85. Springer, Heidelberg (2007)

5. Doucet, A., Godsill, S., Andrieu, C.: On Sequential Monte Carlo Sampling Methods for Bayesian Filtering, Statistics and Computing, pp. 197-208 (2000)

6. Thacker, N.A., Aherne, F.J., Rockett, P.I.: The Bhattacharyya Metric as an Absolute Similarity Measure for Frequency Coded Data. In: TIPR vol. 34, pp. 363-368 (1998)

7. Weisstein, E.W.: Rotation Matrix. From MathWorld-A Wolfram Web Resource 
8. Saff, E., Kuijlaars, A.: Distributing many points on a sphere. The Mathematical Intelligencer 19(1), 5-11 (1997)

9. Suryanarayanan, S., Mullick, R., Mallya, Y., Kamath, V., Nagaraj, N.: Automatic partitioning of head CTA for enabling segmentation. In: Fitzpatrick, J., Sonka, M. (eds.) SPIE Medical Imaging, vol. 5370, pp. 410-419 (2004)

10. Shim, H., Yun, I.D., Lee, K.M., Lee, S.U.: Partition-based extraction of cerebral arteries from CT angiography with emphasis on adaptive tracking. In: Christensen, G.E., Sonka, M. (eds.) IPMI 2005. LNCS, vol. 3565, pp. 357-368. Springer, Heidelberg (2005)

11. Manniesing, R., Viergever, M., Niessen, W.: Vessel axis tracking using topology constrained surface evolution. IEEE Transaction on Medical Imaging 26(3), 309$316(2007)$ 